\title{
Effect of Different Types of Shoes on Lower Limb Muscular Activation during Walking in Women
}

\author{
Bo-Ra Kang ${ }^{1}$, Jin-Gang Her ${ }^{2}$, Ji-Hea Woo ${ }^{3}$, Hee-Soo Kim ${ }^{4}$, Sin-Ho Chung ${ }^{5}$ \\ ${ }^{1}$ Yonseimadu Hospital, Department of Occupational Therapy Republic of Korea, violet781@ nate.com \\ ${ }^{2}$ Graduate School of Hallym University, Department of Rehabilitation Therapy Republic of Korea, jghur7@empas.com \\ ${ }^{3}$ Hallym Polytechnic Department of Physical Therapy University, Republic of Korea, palpation@empas.com \\ ${ }^{4}$ Hallym Polytechnic Department of Physical Therapy University, Republic of Korea, otkimhs@empas.com \\ ${ }^{5}$ Hanyang University Medical Center, Department of Physical Therapy Republic of Korea, wwin72@empas.com
}

*Correspondence author - Jin-Gang Her, PhD; jghur7@empas.com

Received 14 March 2019;

Accepted 22 March 2019;

Published 28 March 2019

\begin{abstract}
Purpose: The present study intended to compare the effects on the female body of high-heeled shoe types, including regular high heels, wedge heels, and platform heels, with those of sneakers during level walking by examining muscular activity in the lower limbs. Materials and Methods: Participants were 20 women in their 20 s or 30 s who regularly wear different types of size 8 (US size). The heel height tested was $7 \mathrm{~cm}$, and electromyographic muscular activation (\% maximal voluntary isometric contraction) was measured in the rectus femoris, tibialis anterior, and semitendinosus muscles of participants' dominant legs during walking $15 \mathrm{~m}$ at a self-selected walking velocity step speed. $\underline{\text { Results: Rectus }}$ femoris revealed significant electromyographic differences among shoe types in terms of muscular activation $(p=.013)$. Multiple comparisons among shoe types and activation of various muscles were carried out. These showed significant $(\mathrm{p}<.05)$ differences in rectus femoris muscular activation among shoe types, most notably between sneakers and platform heels (average $-15.69(\mathrm{SD}=4.22)(\mathrm{p}=.002)$ ) and between sneakers and high heels (average $13.83(\mathrm{SD}=4.22)(\mathrm{p}=.010)$ ). Conclusion: Results obtained revealed significant variation in muscular activation of rectus femoris among shoe types. Multiple pairwise comparisons of the differences in muscular activation of rectus femoris revealed significant differences between sneakers and high heeled shoes and between sneakers and platform heels. Therefore, the results of the present study are expected to be beneficial for ordinary individuals in selecting appropriate types of shoes that reduce the level of the muscle activation.
\end{abstract}

Keyword: Gait, Lower extremity, Maximal voluntary isometric contraction Muscular activation, Shoes

\section{Introduction}

High-heeled shoes fulfill the aesthetic desires of women in modern society by making them appear taller, possibly projecting an impression of authority or independence ${ }^{[1,2,3]}$. A survey conducted with 447 female university students in Korea on their experiences wearing high-heeled shoes showed approximately $85.5 \%$ of respondents had worn high-heeled shoes: $63.1 \%$ reported they wore high-heeled shoes less than once a week; and 5.2\% reported they wear high-heeled shoes almost every day ${ }^{[4]}$. Reportedly, $61 \%$ of female shoes purchased in the Republic of Korea in 2015 had heels over $7 \mathrm{~cm}$, showing their ongoing popularity $^{[5]}$.

However, according to results from previous studies, wearing a high-heeled shoe may cause several problems. High-heeled shoes increase pressure from the wearer's weight on the forepart of the foot, affecting soft tissues of the foot and ankle joints, and changing the contraction patterns of distal muscles, making the ankles less stable ${ }^{[6]}$. Furthermore, high-heeled shoes increase balance thresholds for foot and ankle joints. As a result, they move the head backward to maintain balance in the standing position, change the degree of lumbar curvature, and induce altered posture of normal body posture, such as flexion of hip or knee joints ${ }^{[7]}$. Higher heel height increases the instability of the wearer's body; consequently, increasing the muscle tension and muscle activation are induced. The smaller the sole area in contact with the ground, the greater the effect found on muscular activation, center of body mass, and pressure applied to the soles of the feet ${ }^{[8,9]}$. Further studies explored effects associated with different types of heels and shoes, and these have reported a wider shoe sole area is associated with decreased vertical and anteroposterior range of variation of center of body mass ${ }^{[10]}$. Despite these negative reported effects of high-heeled shoes, the level of preference for high-heeled shoes among women still remains high, and there are varieties of highheeled shoes such as wedge heels or platform heels.

The term "high-heeled shoes" generally refers to shoes with elevated heels; in broader context, the term typically refers to such 
shoes made for women ${ }^{[11]}$. Wedge heels, named after the shape of heel inserted into bottom of shoes like a wedge, have advantages of securing stabilized balance despite the heel height ${ }^{[12]}$. According to the book that is written by shoe professionals, platform heels absorb shocks from the sole in contact with the ground surface through their thick platforms ${ }^{[12,13]}$. However, on uneven surfaces, the thick heel of platform heels may decrease foot and ankle stability and potentially result in more risk of injury than other types of shoes ${ }^{[13]}$. Sneakers are light but sturdy canvas and rubber or leather shoes, typically worn for leisure activities ${ }^{[14]}$. Sneakers designs have employed ergonomic elements alongside factors that promote the sneakers as fashion items ${ }^{[15]}$.

A few studies delving into the general effects on the human body of sneakers and high-heeled shoes with differing shapes of soles and heels have already been conducted, mainly focusing on their area and height. The present study was designed to expand on this limited knowledge and identify the effects of varied shapes of shoes, specifically for women, on the muscular activation of lower limbs.

\section{Material and Method}

The 20 participants who participated in the present study were selected from women in their 20 s or 30 s who wore shoes sized 8 (US size), and who were accustomed to wearing shoes with high heels (over $7 \mathrm{~cm}$ ) more than 4 hours a day, more than 3 days a week $^{[16,17,18]}$. Exclusion criteria were: musculoskeletal disorders; diseases of the lower limbs; history of previous lower extremity operations; ankle injury in the prior 12 months; lower back pain in prior the 12 months; serious deformities in the feet; and neurological disorders.

Shoes employed for the present study were high heels, wedge heels, platform heels, and sneakers (Figure 1). The heel height was set at $7 \mathrm{~cm}$, based on the results of a survey of heel-height preferences conducted with female respondents in their $20 \mathrm{~s}^{[19]}$. The size of shoes chosen for the present study was 8 (US size), the most common size in a survey about sneakers of respondents in their 20 s and 30s, wherein the shoe-size distribution was as follows: size 8 (US size), 26.7\%; size 7.5 (US size), 25.9\%; size 8.5 (US size) $17.0 \%$; and size 7 (US size), $16.6 \%{ }^{[20]}$.

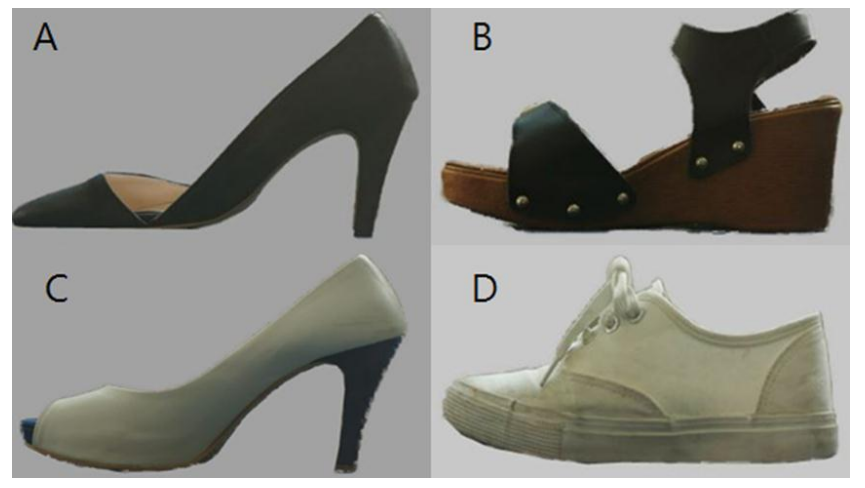

Figure 1: Footwear used in the study: A) High-heel, B) Wedgeheel, C) Platform-heel, D) Sneakers

The participants were assigned to wear the 4 types of shoes in random order; 2 minutes was allowed for adaptation to each pair of shoes before the initiation of each experiment to minimize experimental errors due to maladaptation or muscular fatigue ${ }^{[19]}$. Self-selected step speeds for each participant were allowed for the walking distance of $15 \mathrm{~m}$ used in the experiment ${ }^{[19]}$. Self-selected step speeds checked by using the metronome for consistency across the testing. The dominant leg was selected for EMG during the 15-m walking session; the dominant leg was distinguished by identifying the leg used for kicking a ball ${ }^{[21]}$. The muscles subjected to EMG measurement were the rectus femoris, semitendinosus, and tibialis anterior; the protocols of Surface Electromyography for the Non-Invasive Assessment of Muscles (SENIAM) were referred to for the attachment of adhesive electrodes to skin: The electrode was attached to the point on the center of line that runs from the superior pole of the patella to the Anterior Superior Iliac Spine (ASIS) for the measurement of rectus femoris. For tibialis anterior, the electrode was attached to the point $1 / 3$ of the way down the line segment connecting the head of the fibula to the medial malleolus. The midpoint of the line segment connecting the ischial tuberosity to the medial tibial epicondyle was the placement for the EMG electrode for semitendinosus ${ }^{[22]}$. The attachment of electrodes was done by the same experimenter for all participants to avoid potential errors in EMG measurement due to variations in electrode attachment technique and placement.

A wireless surface electromyography system, the Mini WAVE Infinity Waterproof EMG (Cometa Systems Inc, Milan, Italy), was employed in the present study to measure muscular activation of lower limbs expressed in terms of electromyography (EMG) (Figure 2). Three adhesive dual electrodes of $\mathrm{Ag}-\mathrm{AgCl}$ type, spaced $2 \mathrm{~cm}$ apart, were selected as surface electrodes for the measurement of EMG. The electrodes were attached to each point after epilation and subsequent disinfection with alcohol.

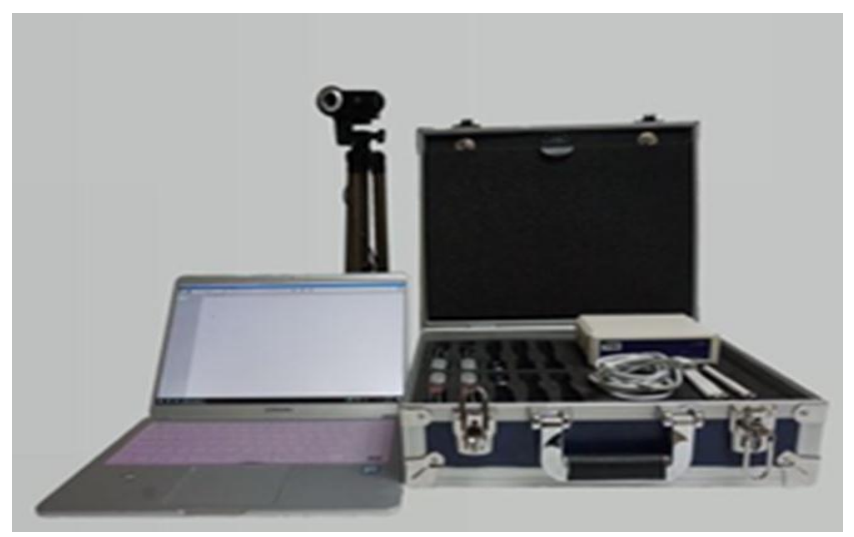

Figure 2: EMG measuring tools

The frequency used for EMG signal sampling was 2,000 $\mathrm{Hz}$ with a band-pass filter of 10-500. To standardize EMG measurements, the percent maximal voluntary isometric contraction (\%MVIC) was used for quantification. EMG measurement was carried out three times. Measurements of muscular activation were collected from participants walking $15 \mathrm{~m}$. The average values of the three measurements were used for the analysis ${ }^{[23]}$. The signals of muscular activity collected from the experiment were converted into Root Mean Square (RMS) figures for the analysis.

\section{Statistical Analysis}

PASW for Windows (IBM/SPSS Inc., Chicago, IL) was used for statistical analyses. To evaluate differences in \%MVIC among the three muscles (rectus femoris, semitendinosus, and tibialis anterior) in relation to the 4 types of shoes, a repeated-measures ANOVA was carried out. The level of statistical significance was 
set at $\alpha=0.05$. The Dunn-Bonferroni procedure was used to conduct multiple comparisons.

\section{Results}

The participants participating in the present study were women in their 20s and 30s; the general characteristics comprising measurements of age, height, weight, and BMI are summarized in Table-1.

Table-1: General characteristics of the participants

\begin{tabular}{|l|c|}
\hline & Mean \pm SD \\
\hline Age (years) & $27.20 \pm 2.60$ \\
\hline Height $(\mathrm{cm})$ & $162.35 \pm 4.94$ \\
\hline Weight $(\mathrm{kg})$ & $55.90 \pm 3.66$ \\
\hline BMI & $21.26 \pm 1.35$ \\
\hline
\end{tabular}

The muscular activities of rectus femoris, semitendinosus, and tibialis anterior, expressed in terms of (\%MVIC) corresponding to each type of shoes, were compared to each other, from which, the rectus femoris was found with significant differences in measurements of muscular activities corresponded to each type of shoes $(\mathrm{F}=3.829, \mathrm{p}=.013)$. However, the semitendinosus and tibialis anterior did not render significant differences in muscular activities corresponded to each type of shoes ( $\mathrm{p}>.05)$ (Table-2).

In respect of multiple comparisons of muscular activities of rectus femoris corresponding to each type of shoes, the average differences in muscular activities resulted from the correspondence of platform heels to sneakers $(-15.69(\mathrm{SD}=4.22)(\mathrm{p}=.002))$ and from the correspondence of sneakers to high heels $(-13.83$ (SD = $4.22)(\mathrm{p}=.010))$ were found statistically significant $(\mathrm{p}<.05)$. However, the rectus femoris did not exhibit significant differences in muscular activities from other types of shoes $(\mathrm{p}>.005)$.

Table 2: Comparison of activation of dominant lower limb muscles in each type of shoes

\begin{tabular}{|l|c|c|c|c|c|c|c|}
\hline \multicolumn{2}{|c|}{} & Sneakers & Wedge heel & Platform heel & High heel & F & p \\
\hline MVIC (\%) & TA & $30.51 \pm 13.70$ & $31.76 \pm 13.70$ & $33.59 \pm 18.87$ & $28.86 \pm 14.39$ & 0.638 & 0.594 \\
& RF & $15.89 \pm 9.45$ & $20.49 \pm 11.27$ & $31.58 \pm 16.86$ & $29.72 \pm 14.59$ & 3.829 & $0.013^{*}$ \\
& ST & $10.07 \pm 3.03$ & $11.39 \pm 3.25$ & $12.16 \pm 3.64$ & $11.31 \pm 3.55$ & 0.234 & 0.872 \\
\hline
\end{tabular}

$* p<.05$, mean \pm standard deviation., TA: Tibialis anterior; RF: Rectus femoris, ST: Semitendinosus

\section{Discussion}

This study aimed to identify patterns of muscular activation visà-vis shoe type in the lower limbs of female participants walking a level distance of $15 \mathrm{~m}$, using an EMG to measure \%MVIC. The 4 types of shoes evaluated were high heels, wedge heels, platform heels, and sneakers. Our goal was to provide women in their $20 \mathrm{~s}$ and 30s with referential information for the selection of shoes. Our hypothesis was that different types of high-heeled shoes (high heels, wedge heels, and platform heels) would affect muscular activation in the lower limbs in different ways in relation to each other, as well as in relation to sneakers.

Thus, three muscles (tibialis anterior, rectus femoris, and semitendinosus) were selected to measure variations in muscular activation according to different shoe types. These three specific muscles were selected for the following reasons: Tibialis anterior suppresses foot drop in the swing phase of walking gait and pulls the tibia forward in the stance phase. Rectus femoris limits knee flexion, along with the other quadriceps femoris muscles, to promote the progression of lower limbs in the stance phase. Semitendinosus, as an extensor of the hip joint and a flexor of the knee joint, bridges the transition from the intermediate swing phase to the terminal swing phase. Thus, these three muscles, involved throughout the swing and stance phases, were selected to capture the full range of muscular activation ${ }^{[24]}$.

Results revealed significant differences in the activation of the rectus femoris corresponding with shoe type. However, the tibialis anterior and semitendinosus showed no significant differences. Therefore, multiple pairwise comparisons of measurements of muscular activity of the rectus femoris in different shoe types were carried out. The results showed significant differences between sneakers and platform heels, and between sneakers and high heels; whereas the pairwise comparisons with other shoe types yielded no significant differences. The results partially agreed with our study hypothesis that measurements of muscular activation of lower limbs would vary according to shoe type. However, differences were not found in muscular activity between wedge heels, platform heels, and high heels.

For rectus femoris, such results have been assessed likely to be attributable to the backward movement of knee joints of participants who wore high-heeled shoes. to maintain balance while wearing new and different heights of heels ${ }^{[25]}$. With the knee joint moving backward, the axis of motion becomes more distant from the line of gravity. This increases the external moment of force straightening the knee joint, and thereby stresses the knee's flexor muscles, followed by a reaction of the muscle spindle reflex that activates the alpha motor neurons, causing an increase in muscular activation of the rectus femoris ${ }^{[18]}$. Thus, wearing highheeled shoes leads to an increase in the muscular activity of the rectus femoris, increasing the anterior shearing force on the knee, readily triggering knee pain ${ }^{[26,27]}$. This lends significance to our finding that the muscular activity of the rectus femoris was increased by wearing high-heeled shoes in the present study.

However, the tibialis anterior and semitendinosus did not show significant differences in muscular activation under conditions of different shoe types. It is notable, however, that tibialis anterior muscular activation levels were higher than those of the other 2 muscles under all test conditions. This likely represents continuous concentric and eccentric contractions of the muscles around the ankle to maintain a standing posture. While our test was too brief to induce fatigue, these results could support the outcomes of previous studies that concluded muscle fatigue would increase due to ankle instability as heel height increased ${ }^{[18,28]}$.

On the other hand, the wedge heels yielded no significant differences in muscular activity from multiple comparisons with different types of shoes, including sneakers, platform heels, and high heels. The results are similar to those of a previous paper wherein the muscular activity of gastrocnemius increased with heel height, regardless of varying the area of heels over widths of $1 \mathrm{~cm}$, $4 \mathrm{~cm}$, and $9 \mathrm{~cm}^{[8]}$. The results can also be compared with the results of another previous study that reported muscular activation in different cervical and lumbar levels varying in different standing 
postures while barefoot and in 3 types of shoes; wedge heel, setback heel, and French heel. This was in contrast to the vertebral erector muscles, which displayed no significant differences in muscular activity among the 3 different types of shoes ${ }^{[29]}$. Closer comparisons associated with the effects of ordinary wedge heels were needed in the present study, however most previous studies have employed wedge heels which were modified for treatment purposes, instead of wedge heels made for ordinary wear ${ }^{[30]}$.

Activity of the rectus femoris revealed significant differences between sneakers and platform heels, and sneakers and high heels. Thus, the results obtained from the present study are expected to be beneficial for women in selecting proper footwear for their daily lives. To benefit the $59 \%$ of women in modern society who wear high-heeled shoes ${ }^{[31]}$, more such studies delving into the effects of diverse kinds of shoes on the female body are needed.

Limitations: In the present study, varied heel heights of shoes affected muscular activation in the lower limbs more than the different shapes of shoes. However, a small number of participants participated in the present study, limiting its validity compared with that of previously conducted studies. Adjustments in data to account for diverse factors affecting the human body, and to compensate for individual differences among participants, were also limited by small sample size ${ }^{[8,32]}$. Based on the results of the present study, future studies to further explore the effects of changes in the shapes of shoe heels on the human body are needed to clarify these issues. Appropriate suggestions to prevent musculoskeletal diseases attributable to the wearing of high-heeled shoes could then be made.

\section{Conclusions}

According to results obtained from the present study, the muscular activity of the rectus femoris, but not semitendinosus or tibialis anterior, was found to have significant variation among different types of shoes. Multiple pairwise comparisons of the muscular activity of the rectus femoris showed significant differences between sneakers and platform heels and between sneakers and high heels. These results are expected to be beneficial for women in selecting appropriate high-heeled shoes for daily wear. Our data also suggests directions for further research into the topic of heel height and muscle activation.

\section{Declarations}

Ethics approval and consent to participate: HIRB-2017-063

\section{Funding}

Not applicable

\section{Competing contributions}

The authors declare that they have no competing interests. The authors have no financial disclosures to declare, no conflicts of interest to report and have no commercial or proprietary interest.

\section{Acknowledgements}

Not applicable

$$
\text { Author contributions }
$$

The authors have no financial disclosures to declare, no conflicts of interest to report and have no commercial or proprietary interest.

\section{References}

[1] McPoli TG Jr. Footwear. Phys Ther 1988; 68(12):185765.

[2] Kim MK, Kim YH, Yoo KT. Effects of shoe type on lower extremity muscle activity during treadmill walking. J Phys Ther Sci 2015;27(12):3833-36.

[3] Iqbal R, De A, Mishra W, et al. Study on lumbar kinematics and the risk of low back disorder in female university students by using shoes of different heel heights. Work 2012; 41(1):2521-26.

[4] Kim SY, Park EJ, Kim HM, et al. Female college student wears high heels. The Korean Public Health; 2011, p. 32.

[5] Kim MH. women, take off high heels-Kumkang shoes, slip-ons and low-heeled shoes sales. Financial News 2015;

http://www.fnnews.com/news/201508111440243440.

[6] Simonsen EB, Svendsen MB, Nørreslet A, et al. Walking on high heels changes muscle activity and the dynamics of human walking significantly. J Appl Biomedch 2012;28(1):20-8.

[7] Gefen A, Megido-Ravid M, Itzchak Y, et al. Analysis of muscular fatigue and foot stability during high-heeled gait. Gait Posture 2002;15(1): 56-63.

[8] Lee CM, Jeong UH. The study on musculoskeletal effects of heel types. J Ergon Soc Korea 2004;23(1):3948.

[9] Chien HL, Lu TW. Effects of shoe heel height on the end-point and joint kinematics of the locomotor system when crossing obstacles of different heights, Ergonomics 2017;60(3): 410-20.

[10] Ferber R, Osternig LR, Woollacott MH, et al. Reactive balance adjustments to unexpected perturbations during human walking. Gait Posture 2002;16(3):238-48.

[11] Wikipedia. High heels. 2018, https://ko.wikipedia.org/wiki/\%ED\%95\%98\%EC\%9D\% B4\%ED\%9E\%90,

[12] Fashion professional dictionary the compilation committee. Fashion professional dictionary. Fashion Korea dictionary research publishing; 2017. p. 302.

[13] SODA life. Women's spring shoes, platform shoes and single $\quad$ soles. 2017, https://blog.naver.com/dfd_soda/220969076150.

[14] Britannica Visual Dictionary. Running shoes. 2012;http://www.ikonet.com/en/visualdictionary/static/us /searchresults?cx=partner-pub7156007925185226\%3Airtdbf$4 \mathrm{~s} 1 \mathrm{w} \& \mathrm{cof}=$ FORID $\% 3 \mathrm{~A} 9 \& \mathrm{ie}=\mathrm{UTF}-$ $8 \& q=$ Running+shoes\&siteurl=www.ikonet.com $\% 2 \mathrm{Fen} \%$ 2Fvisualdictionary $\% 2 \mathrm{~F} \& \mathrm{ref}=\mathrm{www}$. ikonet.com $\% 2 \mathrm{Fen} \% 2$ F\&ss=2149j1569409j5.

[15] Namuwiki. Running shoes. 2018; https://namu.wiki/w/\%EC\%9A\%B4\%EB\%8F\%99\%ED $\% 99 \% 94$.

[16] Yun SY. Changes of lumbar lordosis caused by different heel heights in healthy women. Physical Therapy Korea 1999;6(2):43-55.

[17] Lee C. The effects of lower extremity angle according to heel-height changes in young ladies in their 20s during gait. J Phys Ther Sci 2014;26(7):1055-58. 
[18] Snow RE, Williams KR. High heeled shoes: their effect on center of mass position, posture, three-dimensional kinematics, rearfoot motion, and ground reaction forces. Arch Phys Med Rehabil 1994;75(5)568-76.

[19] Kim SG, Im DH, Kong EK. Muscle activation change of rectus abdominal muscle and erector spinae muscle depending on shoe heel-height on gait. JKPTS 2012;19(2):1-7.

[20] Onpanel Korea. Survey of sneakers usage and preference. 2013; https://www.onpanel.co.kr/mergeboard/board/boardView ?am_seq=\&site_code $=32 \&$ skin_change $=\&$ layout $=$ defaul t_sub\&board_id=dataroom_market\&search_key=\&searc h_val $=\&$ select $1=\&$ select $2=\&$ sel_category $=\&$ catecode $=\&$ site_type $=$ onpanel\&pagecnt $=15 \&$ write_seq $=18$.

[21] Oh DW, Kim ST. Effect of shoe heel height on vastus medialis and vastus lateralis electromyographic activity during stair ascending and descending. Physical Therapy Korea 2009;16(3):24-31.

[22] Hermens HJ, Freriks B. Surface electromyography for the non-invasive assessment of muscles. 2006; http://www.seniam.org.

[23] S. M. Kim and T. S. Ko, "The effect of muscle activity in tibialis anterior \& gastrocnemius when women ascend the stairs with different height of high-heel", The Korean Society of Medicine \& Therapy Science, vol. 8, no. 1, pp. 21-28, 2016.

[24] Perry J, Burnfield JM. Gait analysis: Normal and pathological function 2 nd edition. SLACK publisher;2010.
[25] Opila KA, Wagner SS, Schiowitz S, et al. Postural alignment in barefoot and high-heeled stance. Spine 1988;13(5):542-7.

[26] Neymann DA. Kinesiology of the musculoskeletal system: foundations for physical rehabilitation. St Louis: Mosby publisher;2009.

[27] Sahrmann SA. Diagnosis and treatment of movement impairment syndromes. St Louis: Mosby publisher; 2002.

[28] Svantesson U, Osterberg U, Thomee R, et al. Muscle fatigue in a standing heel-rise test. Scand J Rehabil Med 1998;30(2):67-72.

[29] Han D. Muscle activity of paraspinal muscles in different types of high heels during standing. JPTS 2015;27(1):679.

[30] Wolfe SA, Brueckmann FR. Conservative treatment of genu valgus and varum with medial/lateral heel wedges. Indiana med 1991;84(9): 614-5.

[31] Yu J, Cheung JT, Fan Y, et al. Development of a finite element model of female foot for high-heeled shoe design. Clin Biomech 2008;23(1):31-8.

[32] Kim HS, Kim HT, Park JH. A study of mathematical human modeling of sitting crew during whole-body vibration. J Ergon Soc Korea 2003;22(1):1-15.
Abbreviations
MVIC: Maximal voluntary isometric contraction
TA: Tibialis anterior
RF: Rectus femoris
ST: Semitendinosus 